\title{
A prospective study of tracheal intubation in an academic emergency department in Malaysia
}

\author{
Shahridan Mohd. Fathil • Siti Nidzwani Mohd. Mahdi • \\ Zuraidah Che'Man • Azhana Hassan • \\ Zulkernain Ahmad • Ahmad Khaldun Ismail
}

Received: 21 December 2009 /Accepted: 13 June 2010/Published online: 21 September 2010

(C) Springer-Verlag London Ltd 2010

\begin{abstract}
Background Airway management is an important part of the management of the critically ill and injured patients in the Emergency Department (ED). Numerous studies from developed countries have demonstrated the competency of emergency doctors in intubation. To date there have been no published data on intubations performed in EDs in Malaysia. Methods Data on intubations from 7 August 2007 till 28 August 2008 were prospectively collected.

Results There were 228 intubations included in the study period. Cardiopulmonary arrest was the main indication for intubation $(35.5 \%)$. The other indications were head injury $(18.4 \%)$, respiratory failure $(15.4 \%)$, polytrauma $(9.6 \%)$ and cerebrovascular accident (7.0\%). All of the 228 patients were successfully intubated. Rapid sequence intubation (RSI) was the most frequent method (49.6\%) of intubation. A total of $223(97.8 \%)$ intubations were done by ED personnel. In $79.8 \%$ of the cases, intubations were successfully performed on the first attempt. Midazolam was the most common induction agent used (97 patients), while suxamethonium was the muscle relaxant of choice (109 patients). There were 34 patients (14.9\%) with 38 reported immediate complications. The most common complication was oesophageal intubation.
\end{abstract}

Disclaimer The views expressed in this paper are those of the author (s) and not those of the editors, editorial board or publisher.

S. M. Fathil $(\bowtie) \cdot$ S. N. Mohd. Mahdi · Z. Che'Man · Z. Ahmad ·

A. K. Ismail

Emergency Department,

Universiti Kebangsaan Malaysia Medical Centre,

56000 Kuala Lumpur, Malaysia

e-mail: cooldoc1971@yahoo.co.uk

A. Hassan

Emergency and Trauma Department, Hospital Kuala Lumpur,

Kuala Lumpur, Malaysia
Conclusion Emergency Department UKMMC personnel have a high competency level in intubation with an acceptable complication rate. RSI was the most common method for intubation.

Keyword Airway · Intubation - Rapid sequence intubation · Orotracheal intubation

\section{Introduction}

In Malaysia, Emergency Medicine (EM) is a newly recognised specialty with the first postgraduate program starting in 1998 [1]. Emergency Department Universiti Kebangsaan Malaysia Medical Centre (ED UKMMC) started the EM postgraduate program in 2005. UKMMC is an 800-bedded university hospital. The emergency department is made up of various levels of health care professionals consisting of emergency specialists (emergency physicians, anaesthesiologists and orthopaedic surgeons), non-specialist service medical officers, EM post-graduate trainees, paramedics and nurses. All ED UKMMC resuscitations were managed using a team approach according to recognised international guidelines (Advanced Cardiac Life Support, Paediatric Advanced Life Support and Advanced Trauma Life Support). Besides the compulsory life-support training, the department also provides in-house airway training in the form of manikin simulation. The postgraduate trainees in EM also undergo a 6-month anaesthetic posting during their first year of training.

Airway management is an important part of the management of the critically ill and injured in the Emergency Department (ED). Rapid and safe control of the airway is vital to maintain adequate oxygenation. Numerous studies from developed countries have demon- 
strated the competency of emergency doctors in establishing a definitive airway [2-11] in their settings, but there is currently no published data on definitive ED airway management in Malaysia.

\section{Objectives}

The general objective of this study was to describe the current practice of definitive airway management in the ED UKMMC. The specific objectives were to determine the success rates of endotracheal intubation and to describe the immediate complications.

\section{Materials and methods}

This was an observational study performed in the ED UKMMC from 7 August 2007 till 28 August 2008. Approval of the institutional Independent Review Board was obtained prior to data collection.

The inclusion criterion was patients undergoing endotracheal intubation in ED UKMMC. The exclusion criteria were pre-intubated patients transferred from other hospitals or other places beside the ED (i.e., wards or clinics), any missed documentation of intubation events and incomplete data.

A standardised form was filled in by ED health care professional after performing endotracheal intubation. Completed forms were collected on a daily basis. The Triage 1 registry was reviewed for any missed documentation of intubation events. These data were acknowledged but not included in the final analysis.

The information collected was patient age, sex, estimated weight, indication for intubation, number of attempts, technique of definitive airway management, all medications used to facilitate intubation and immediate complications of intubations. The level of training and specialty of the professional performing the intubation were also documented.

For the purpose of this study, methods of airway management were initially classified into orotracheal intubation (OTI), rapid sequence intubation (RSI), nasotracheal intubation (NTI) and cricothyrodotomy (CR). Orotracheal intubation (OTI) was defined as oral intubation using direct laryngoscopy with or without the use of a sedative agent. Rapid sequence intubation (RSI) was defined as oral intubation using direct laryngoscopy with the use of sedative and rapid onset neuromuscular blocking agent to facilitate intubation. Cricothyrodotomy was defined as emergency incision through the skin and cricothyroid membrane to secure the airway.

ED UKMMC maintains different types and sizes of laryngoscope blades (Magill and Macintosh), varying sizes of endotracheal tubes and Classic laryngeal masks (LMA) as standard equipment for both adult and paediatric airway management. Difficult airway adjuncts are available for use. These include the McCoy laryngoscope, intubating LMA, Proseal LMA, gum elastic bougie, flexible fibreoptic scope, and instruments for cricothyrodotomy and transtracheal jet ventilation. Intravenous anaesthetic agents and muscle relaxants available are midazolam, propofol, etomidate, sodium thiopentone, ketamine, suxamethonium and rocuronium.

An attempt at intubation was defined as placement of a laryngoscope into the patient's airway followed by the insertion of an endotracheal tube. The methods for confirmation of endotracheal intubation were direct visualisation of an endotracheal tube passing through the vocal cords and five-point auscultation. Carbon dioxide detecting devices were not available during the study period. Difficult intubation for this study was defined as either an intubation requiring more than two attempts or the usage of alternative intubation techniques.

Immediate complications post-intubation include significant changes in the physiological status and techniquerelated events. Desaturation was defined as a decrease in oxygen saturation by pulse oximetry to less than $90 \%$. Hypotension was defined as a drop in systolic blood pressure to less than $90 \mathrm{mmHg}$ that could not be accounted for by other mechanisms (e.g., acute haemorrhage) or any reduction more than $20 \%$ from the baseline. Endobronchial intubation was recorded to have occurred if the chest radiograph indicated that the tip of the endotracheal tube was in a mainstem bronchus or when the air entry to both lungs equalised after readjusting endotracheal tube level. A pneumothorax was considered to be a complication if it was identified on a post-intubation chest radiograph in the absence of chest trauma to the affected side. Witnessed regurgitation of gastric contents during intubation was taken as vomiting. Any damage to the teeth that was attributable to laryngoscope use was considered as intubation-associated dental trauma.

\section{Results}

During this study period, the ED UKMMC received a total of 70,356 patients, 12,712 (18.1\%) of whom were admitted. The proportion of patients requiring Triage 1 (the highest priority for care) was $1,506(2.1 \%)$, with 374 patients intubated in ED UKMMC during the 12-month and 3-week study period. Only 302 intubations were recorded prospectively; however, only 228 patients had complete documentation based on the study protocol.

Table 1 summarises the demographic characteristics of the patients in terms of age and their main condition for 
Table 1 Demographic characteristics of intubated patients

\begin{tabular}{llll}
\hline Age (years) & Number of patients (\%) & \multicolumn{2}{l}{ Number of patients } \\
\cline { 3 - 4 } & & Medical disorders & Trauma \\
\hline$\leq 1$ & $10(4.4)$ & 10 & 0 \\
$>1-12$ & $5(2.2)$ & 1 & 4 \\
$13-17$ & $7(3.1)$ & 3 & 4 \\
$18-64$ & $149(65.4)$ & 93 & 56 \\
$\geq 65$ & $57(25.0)$ & 54 & 3 \\
Total & 228 & 161 & 67 \\
\hline
\end{tabular}

admission, i.e., medical or trauma. The ages of the patients ranged from newborns to 93 year olds. The mean age of patients intubated was 46.7 years (CI 43.81-49.58) years. The ratio of trauma to medical cases in the study sample was 1: 2.4 . The paediatric population represented $4.4 \%$ $(n=10)$ of the total number of intubated patients.

Table 2 summarises the main indications for intubation. Cardiopulmonary arrest was the main reason for intubation, followed by head injury. Rapid sequence intubation was the method of choice in patients with head injury and respiratory failure. No patients underwent emergency cricothyrodotomy or nasotracheal intubation.

Of the 228 intubated cases, $79.8 \%$ were successfully done on the first attempt, while $17.5 \%$ and $2.6 \%$ were successfully performed on the second and third attempts, respectively. Table 3 summarises the level of training and specialties of the health care professionals intubating and their success rates. There appears to be no apparent difference in the success rates among the non-specialist ED health care professionals.

Table 4 shows that midazolam was the most common induction agent used, followed by propofol. Suxamethonium was the most preferred muscle relaxant. Fentanyl was used as a co-induction agent in 92 patients.

Table 5 summarises the immediate complications of intubation in 34 patients. In these 34 patients, there were
38 immediate adverse events. Of these, 33 patients were managed by ED personnel, and 1 patient was intubated by an anaesthetic trainee. The most common complication was oesophageal intubation, all cases of which were immediately recognised and interventions carried out. This was followed by desaturation, hypotension and endobronchial intubation.

Of 228 intubated patients, 106 (46.5\%) were admitted to critical care units, which comprised of the general intensive care unit, cardiac care unit, paediatric intensive care unit and burns care unit. Twenty patients $(8.8 \%)$ were transferred to the ward, while $6(2.6 \%)$ patients had to be transferred out to other hospitals due to inavailability of critical care beds in UKMMC. Ventilation of patients in the general ward was acceptable at UKMMC because of the limited availability of critical care beds. These patients were generally very ill with poor prognoses. There were 96 (42\%) deaths within $24 \mathrm{~h}$ of admission; 67 of these patients arrived at the ED with cardiac arrest.

\section{Discussion}

There was no published literature available describing ED intubation practices in Malaysia. In this study, the mean age of the medical patients was 56 years \pm 26 , while for trauma patients it was 29 years \pm 21 . The mean age of our trauma patients was consistent with the general data on national trauma-related injury [12]. There was an apparent difference in the mean age of intubated patients in our study (46.7 years), which is less compared to that of other Asian countries, e.g., Hong Kong (60.7 years) and Singapore (63 years) $[7,11]$.

All the patients in this study except for five were intubated by ED doctors and paramedics. The five patients were one neonate, one trauma and three medical-related cases. In the neonatal case, an urgent request for assistance was made to the neonatal intensive care doctor as the ED doctors on duty were inexperienced in performing intuba-
Table 2 Indications for intubation and the different methods of intubation: RSI, OTI and OTI with sedation

\begin{tabular}{llllll}
\hline Indication for intubation & Frequency (\%) & OTI & OTI with sedation & RSI \\
\hline Medical & Cardiopulmonary arrest & $81(35.5 \%)$ & 81 & 0 & 0 \\
& Respiratory failure & $35(15.4 \%)$ & 3 & 6 & 26 \\
& CVA/ICH & $16(7.0 \%)$ & 1 & 0 & 15 \\
& Severe sepsis/septic shock & $14(6.1 \%)$ & 2 & 4 & 8 \\
& Poisoning & $13(5.7 \%)$ & 0 & 3 & 10 \\
\multirow{3}{*}{ Trauma } & Status epilepticus & $2(0.9 \%)$ & 0 & 0 & 2 \\
& Head injury & $42(18.4 \%)$ & 4 & 3 & 35 \\
& Polytrauma & $22(9.6 \%)$ & 8 & 0 & 3 \\
& Burn/inhalation injury & $3(1.3 \%)$ & 0 & 0 & 113 \\
\hline
\end{tabular}


Table 3 Health care professionals who performed intubations in ED UKMMC

\begin{tabular}{lllll}
\hline Health care professional & $\begin{array}{l}\text { Number of successful } \\
\text { intubations }\end{array}$ & $\begin{array}{l}\text { Total number } \\
\text { of attempts }\end{array}$ & $\begin{array}{l}\text { Percentage of } \\
\text { successful 1st } \\
\text { attempts (\%) }\end{array}$ & $\begin{array}{l}\text { Final success rate } \\
\text { in percentage (\%) }\end{array}$ \\
\hline Service medical officers & 87 & 112 & 77.0 & 77.7 \\
EM trainees & 110 & 135 & 80.6 & 81.5 \\
Emergency specialists & 16 & 16 & 100 & 100 \\
Doctors from other specialties & 5 & 5 & 100 & 100 \\
Paramedics & 10 & 12 & 83.3 & 83.3 \\
Total & 228 & 280 & & \\
\hline
\end{tabular}

tion. In the trauma patient, as the trauma team was activated early in the resuscitation efforts, the attending anaesthetist performed the intubation. In one medical case, the patient had a terminal condition and was deemed unsuitable for intubation and active resuscitation by the ED doctors. However, the medical consultant decided to intubate the patient with the assistance of the anaesthesia team. Another medical patient who was deemed to have a difficult airway because of limited neck mobility was also intubated by the anaesthetist electively. The third medical patient was intubated by the anaesthetist colleague because the ED doctors were occupied with other major resuscitation cases.

Data from United Kingdom (UK) surveys indicate a significant prevalence of anaesthetist-performed RSIs, although there was an increasing trend of emergency physician-performed RSIs [4, 9, 10]. In a 1-year survey carried out from 1995-1996, emergency residents and physicians in a single United States (US) ED performed 93\% of intubations with minimal immediate complications [3]. In a university hospital in Glasgow, UK, only $31 \%$ of RSI in EDs were undertaken by emergency physicians, while the majority were still being performed by anaesthetists in 1999. Intubation rates in the same hospital by Emergency Physicians increased to $75 \%$ in 2003, and the complication rates decreased over the 5 years [4]. In a smaller survey in the UK published in 2001, emergency

Table 4 Agents used for intubation

\begin{tabular}{lll}
\hline Name of agent & \multicolumn{2}{l}{ Frequency } \\
\cline { 2 - 3 } & RSI & OTI with sedation \\
\hline Suxamethonium & 109 & \\
Rocuronium & 6 & 11 \\
Midazolam & 86 & 2 \\
Propofol & 13 & 1 \\
Etomidate & 11 & \\
Thiopentone & 8 & 1 \\
Diazepam & 3 & 6 \\
Fentanyl & 86 & \\
\hline
\end{tabular}

physicians performed $26 \%$ of RSIs with minimal complication rates [5].

In a study conducted in a single US ED, success rates of intubation of trauma patients by emergency physicians were similar to those of anaesthetists [8]. A 2-year study from 1999 to 2001 conducted in EDs of seven Scottish teaching hospitals revealed that anaesthetists achieved more good views at laryngoscopy with higher initial success rates during RSI than emergency physicians [9]. A study done in an ED of a district general hospital in Scotland from 2003 to 2006 demonstrated similar findings [10].

This study revealed that the success rate of intubation on first attempts by EM trainees in ED UKMMC was $80.6 \%$, which is slightly lower than a study done by Sagarin on North American EM residents (83\%) [6]. Six patients (2.6\%) were successfully intubated on the third attempt. None required more than three attempts. The percentages of successful intubation on the third or more attempt in Hong Kong, Singapore and Thailand were 5\%, 4\% and 1.9\%, respectively $[7,11,13]$. In one of our six patients with a difficult intubation, a Cormack Lehane (CL) I view was documented in which the initial two unsuccessful attempts were attributed to excessive external laryngeal pressure using backward, upward and right position manoeuvres (BURP), obliterating the view. In another patient, an improvement in the CL view to grade II was documented after repositioning. A gum elastic bougie was used in one of the two patients with a CL grade III view, while the other was successfully intubated with the blind oral intubation

Table 5 Immediate complications

\begin{tabular}{ll}
\hline Event & Frequency \\
\hline Cardiac arrest & 0 \\
Dental and oral trauma & 2 \\
Desaturation & 10 \\
Oesophageal intubation & 15 \\
Hypotension & 8 \\
Endobronchial intubation & 3 \\
Total & 38 \\
\hline
\end{tabular}


technique. Two patients with a CL grade IV view were intubated with a gum elastic bougie and intubating LMA, respectively. The patient who was finally intubated with an intubating LMA was obese, weighing about $100 \mathrm{~kg}$.

In this study, most of the patients were intubated using RSI (49.5\%), followed by OTI without any pharmacological agents (43.4\%). This OTI figure was higher compared to those reported in two different studies in North America $(11 \%$ and $15.2 \%)[3,6]$. However, the OTI rate in Hong Kong was comparable (111 out of 214 patients) [7]. This may be due to the fact that nearly all of the OTIs in our centre were performed in cases of cardiopulmonary resuscitation.

The pharmacological agent most commonly used for RSI and OTI with sedation was midazolam. The other anaesthetic agents, such as propofol and etomidate, were more frequently used by EM trainees than by service medical officers. This may be explained by the fact that EM trainees were exposed to a variety of anaesthetic agents during the 6-month anaesthetic/intensive care rotation in the first year of training. The muscle relaxant of choice in RSI was suxamethonium, which is the standard practice worldwide $[3,7]$.

The actual complication rate in our series was $14.9 \%$ (38/228), which was higher than the $9.3 \%$ rate observed in the US [3] and $10 \%$ in a Hong Kong survey [7]. The most common documented complications were oesophageal intubation, desaturation and hypotension, which were similar to those observed in the UK, North America and Hong Kong [3, 6, 7].

\section{Limitations}

There were several limitations to this study. First, there was difficulty getting the health care personnel to comply with the documentation requirement of the intubation processes in the standard form, which include incomplete documentation of physiological parameters of pre- and post-intubation events. This resulted in 146 out of 374 (39\%) patients being excluded from the analysis because of missing or incomplete data. The second limitation was that most of the data collected were based on reports from different levels of health care professionals, thus contributing to bias.

\section{Conclusion}

Emergency Department UKMMC personnel have high competency levels in intubation with acceptable complication rates. RSI was the most common method for intubation.
Conflicts of interest The authors report no conflict of interest or sources of financial support involved with this manuscript. The authors are responsible for the content and writing of this paper.

\section{References}

1. Rahman NHNA, Holliman CJ (2005) Emergency medicine in Malaysia Hong Kong. J Emerg Med 12(4):246-252

2. Taryle DA, Chandler JE, Good JT et al (1979) Emergency room intubations - complications and survival. Chest 75:541-543

3. Sakles JC, Laurin EG, Rantapaa AA (1998) Airway management in the emergency department: a 1-year study of 610 tracheal intubations. Ann Emerg Med 31(3):325-332

4. Simpson J, Munro PT, Graham CA (2006) Rapid sequence intubation in the emergency department: 5 year trends. Emerg Med J 23:54-56

5. Butler JM, Clancy M, Robinson N et al (2001) An observational survey of emergency department rapid sequence intubation. Emerg Med J 18:343-348

6. Sagarin MJ, Barton ED (2005) Airway management by US and Canadian emergency medicine residents: a multicenter analysis of more than 6,000 endotracheal intubation attempts. Ann Emerg Med 46(4):328-336

7. Tam AY, Lau FL (2001) A prospective study of tracheal intubation in an emergency department in Hong Kong. Eur J Emerg Med 8(4):305-310

8. Bushra JS, McNeil B, Wald DA (2004) A comparison of trauma intubations managed by anesthesiologists and emergency physicians. Acad Emerg Med 11(1):66-70

9. Graham CA, Beard D, Oglesby AJ, Thakore SB, Beale JP, Brittliff J, Johnston MA, McKeown DW, Parke TRJ (2003) Rapid sequence intubation in Scottish urban emergency departments. Emerg Med J 20:3-5

10. Stevenson AGM, Graham CA, Hall R, Korsah P, Mc Guffie AC (2007) Tracheal intubation in the emergency department: the Scottish district hospital perspective. Emerg Med J 24:394-397

11. Wong E, Fong YT, Ho KK (2004) Emergency airway managementexperience of tertiary hospital in south-east Asia. Resuscitation 61:349-355

12. National Trauma Database January to December 2007 Second Report Ministry of Health www.acrm.org.my/ntrd

13. Wongyinnsinn M, Songarj P, Assawivinijkul T (2009) A prospective observational study of tracheal intubation in a emergency department in a 2,300-bed hospital of a developing country in a 1year period. Emerg Med J 26:604-608

Shahridan Mohd. Fathil qualified as a doctor from the University of Malaya, Malaysia, in 1996 and worked in Malaysia until 2000. In 2001, he left to train in anaesthesia in Ireland and England. He obtained his Felllowship of the Royal College of Anaesthetists qualification in 2003. He returned to Malaysia to work in Hospital Kuala Lumpur as an anaesthetic clinical specialist from 2004 until 2005. His next and current position was clinical specialist in the Department of Emergency Medicine, Universiti Kebangsaan Malaysia Medical Centre, where he functions as both anaesthetist and emergency physician. From mid 2007 till mid 2008, he took a sabbatical leave to pursue a Regional Anesthesia Fellowship in the Department of Anaesthesia and Pain Medicine, Royal Perth Hospital, Australia. His interests are regional anaesthesia, resuscitative medicine and critical ultrasound. 